\title{
Dimethylarginine dimethylaminohydrolase 2 promotes tumor angiogenesis in lung adenocarcinoma
}

\author{
Toshihiro Shiozawa $^{1,2} \cdot$ Shinji Iyama $^{1} \cdot$ Shotaro Toshima $^{1} \cdot$ Akiko Sakata $^{1}$. \\ Shingo Usui ${ }^{3} \cdot$ Yuko Minami $^{1} \cdot$ Yukio Sato $^{4} \cdot$ Nobuyuki Hizawa $^{2} \cdot$ Masayuki Noguchi $^{1}$
}

Received: 27 July 2015 /Revised: 28 August 2015 / Accepted: 29 September 2015 /Published online: 29 October 2015

(C) The Author(s) 2015. This article is published with open access at Springerlink.com

\begin{abstract}
Although embryonal proteins have been used as tumor marker, most are not useful for detection of early malignancy. In the present study, we developed mouse monoclonal antibodies against fetal lung of miniature swine, and screened them to find an embryonal protein that is produced at the early stage of malignancy, focusing on lung adenocarcinoma. We found an antibody clone that specifically stained stroma of lung adenocarcinoma. LC-MS/MS identified the protein recognized by this clone as dimethylarginine dimethylaminohydrolase 2 (DDAH2), an enzyme known for antiatherosclerotic activity. DDAH2 was found to be expressed in fibroblasts of stroma of malignancies, with higher expression in minimally invasive adenocarcinoma (MIA) and invasive adenocarcinoma than in adenocarcinoma in situ (AIS). Moreover, tumors with high stromal expression of DDAH2 had a poorer prognosis than those without. In vitro analysis showed that DDAH2 increases expression of endothelial nitric oxide synthase (eNOS), inducing proliferation and capillary-like tube formation of vascular endothelial cells.
\end{abstract}

Electronic supplementary material The online version of this article (doi:10.1007/s00428-015-1863-z) contains supplementary material, which is available to authorized users.

Masayuki Noguchi

nmasayuk@md.tsukuba.ac.jp

1 Department of Pathology, Faculty of Medicine, University of Tsukuba, 1-1-1 Tennodai, Tsukuba, Ibaraki 305-8575, Japan

2 Department of Pulmonary Medicine, Faculty of Medicine, University of Tsukuba, Tsukuba, Ibaraki, Japan

3 Department of Thoracic Surgery, NHO Ibarakihigashi National Hospital, Tokai, Ibaraki, Japan

4 Department of Thoracic Surgery, Faculty of Medicine, University of Tsukuba, Tsukuba, Ibaraki, Japan
In resected human tissues, eNOS also showed higher expression in invasive adenocarcinoma than in AIS and normal lung, similarly to DDAH2. Our data indicate that expression of DDAH2 is associated with invasiveness of lung adenocarcinoma via tumor angiogenesis. DDAH2 expression might be a prognostic factor in lung adenocarcinoma.

Keywords DDAH2 $\cdot$ Angiogenesis · Adenocarcinoma · Malignant stroma $\cdot$ Prognosis

\section{Introduction}

Lung cancer is the leading cause of cancer-related death worldwide. Because most patients are diagnosed at an advanced stage, the prognosis of lung cancer remains poor. Lung adenocarcinoma is the most frequent histological type of lung cancer in Japan and is increasing in the USA and most European countries $[1,2]$.

Recently, a multidisciplinary classification of lung adenocarcinoma has been published, in which several new concepts, such as adenocarcinoma in situ (AIS) and minimally invasive adenocarcinoma (MIA), have been newly defined [3, 4]. AIS shows replacement growth of tumor cells along alveolar structures (lepidic growth) and contains no invasive component. Although MIA has been added to the invasive adenocarcinoma category, its area of invasion is very limited and it is considered as very early stage adenocarcinoma. Therefore, it has been suggested that MIA has a prognosis as favorable as that of AIS. AIS and MIA are usually detected accidentally or by CT screening, and it is assumed that their biological characteristics are different from those of overt invasive adenocarcinoma. The current classification has focused interest on the concept of stepwise progression of peripheral type adenocarcinoma [5]. It is very important to compare the biological 
features of AIS and MIA with those of overt invasive adenocarcinoma, in order to clarify molecular mechanisms involved in malignant progression of lung adenocarcinoma.

With regard to the detection and diagnosis of lung adenocarcinoma by immunohistochemistry, various tumor biomarker proteins have been reported. These biomarker proteins are classified into several types according to their characteristics and include oncogenic, tissue- and/or cell-specific, and embryonic proteins. Epidermal growth factor receptor (EGFR), Her2, B-raf, Ret, Ros and anaplastic lymphoma kinase (ALK) are examples of oncogenic biomarkers associated with prognosis [6-8]. The expression status of these oncogenes also has an important bearing on the selection of targeted therapeutic drugs, since various types of tyrosine kinase inhibitors are available. On the other hand, tissue- and/or cell-specific biomarkers include cyto-keratins (AE1/AE3, CK7, CK20, etc.), membrane markers of lymphocytes (CD20, CD79, CD3, CD4, CD8, CD9, etc.) and differentiation-specific markers such as TTF-1 for alveolar epithelial differentiation. Although tissue- and/or cell-specific biomarkers are suitable for differential diagnosis between cancer and lymphoma, or between lung adenocarcinoma and metastatic adenocarcinoma, they are not useful as indicators of malignancy.

Although it is noteworthy that these tumor biomarkers include embryonic and fetal proteins such as carcinoembryonic antigen (CEA), OCT3/4, and SOX2 [9-11], few attempts have been made to search for fetal biomarkers systematically. Experimental use of human fetal tissue has various associated ethical issues. Fortunately, most antibodies against swine tissue cross-react with human tissue, since swine messenger RNA (mRNA) has more than $80 \%$ homology with its human counterpart. In view of this homology, fetal swine tissue constitutes an ideal antigenic material for producing antibodies reactive with human fetal tissue and human cancer tissue as embryonic biomarker.

In the present study, we immunized mice against fetal swine tissue and produced monoclonal antibodies (mAbs) reactive with human lung adenocarcinoma. One reacted characteristically with the stromal region of human lung adenocarcinoma, and the antigen was identified as dimethylarginine dimethylaminohydrolase 2 (DDAH2) by LC/MS-MS analysis. We demonstrate that DDAH2 is a marker of tumor angiogenesis and is expressed in lung adenocarcinoma at an early stage.

\section{Materials and methods}

\section{Patient selection}

We selected 133 cases of lung adenocarcinoma that had been resected at Tsukuba University Hospital (Ibaraki, Japan) between 2002 and 2013. All patients had given informed consent for study of their materials before collection. The adenocarcinomas were classified according to the World Health Organization (WHO) classification (fourth edition) and the UICC TNM classification of malignant tumors (seventh edition) [12]. The patients' clinicopathological characteristics are summarized in Supplementary Table 1.

\section{Production of monoclonal antibodies}

The normal length of gestation period in swine is approximately 16 weeks. So, we selected normal fetuses of CLAWN strain miniature swine (Japan Farm CLAWN Institute, Kagoshima, Japan) at weeks 7 and 13 of gestation, and they were harvested surgically from maternal swine. Fetal lungs were resected and embedded in Tissue-Tek OCT Compound (Sakura Finetek Japan, Tokyo, Japan), quickly frozen, and stored at $-80{ }^{\circ} \mathrm{C}$. Protein extraction was performed using T$\mathrm{PER}^{\mathrm{TM}}$ Tissue Protein Extraction Reagent (Thermo Scientific, Rockford, IL) with protease inhibitor cocktail (Sigma-Aldrich, St. Louis, MO). Protein concentration was determined using a bicinchoninic acid protein assay (Thermo Scientific) with bovine serum albumin as the standard. Antigen was prepared as a mixture of extracted protein and an equivalent amount of complete Freund's adjuvant (CFA) (Sigma-Aldrich). CFA/antigen emulsion was injected subcutaneously into the bilateral footpad of a 5-week-old BALB/c mouse on day 1 , and again on day 10 . On day 14 , bilateral popliteal lymph node cells were harvested and fused with the murine myeloma cell line Sp2/0 using polyethylene glycol. Hybridomas were selectively cultured and cloned using the methylcellulose method in a $10 \%$ incubator at $37^{\circ} \mathrm{C}$. Fusion, selection, and cloning were performed with a ClonaCell ${ }^{\circledR}$-HY Hybridoma Cloning Kit (Stemcell Technologies, Vancouver, British Columbia, Canada) in accordance with the manufacturer's protocol. The isolated hybridoma colonies were each suspended in individual wells of a 96-well plate.

\section{Antigen characterization}

The immunoglobulin isotype and subtype of the selected hybridoma clone was determined using a Mouse Monoclonal Antibody Isotyping Test Kit (AbD Serotec, Kidlington, UK).

A lung adenocarcinoma cell line (Calu-3) was used for Western blotting and immunoprecipitation (IP). Calu-3 was purchased from the American Type Culture Collection (Manassas, VA). Total protein was extracted from Calu-3 using MPER mammalian protein extraction reagent (Thermo Scientific) and from frozen tissue of surgically resected lung adenocarcinoma with T-PER. Extracted proteins were denatured at $95{ }^{\circ} \mathrm{C}$ for $5 \mathrm{~min}$, separated by SDS polyacrylamide gel electrophoresis (SDS-PAGE) under reducing conditions, and transferred to PVDF membranes using an iBlot Gel Transfer System (Life Technologies Japan, Tokyo, Japan). After 
blocking, the membranes were incubated with purified monoclonal antibody $(2 \mu \mathrm{l} / \mathrm{ml})$ as the primary antibody for $1 \mathrm{~h}$ at room temperature. Antigen protein was detected using HRPconjugated anti-mouse Igs (Thermo Scientific), and the protein bands were visualized with SuperSignal West Dura extended duration substrate (Thermo Scientific) and X-ray film (Kodak, Rochester, NY).

Antigen protein that had reacted with the selected monoclonal antibody was immunoprecipitated from extracted total protein of frozen lung adenocarcinoma tissue with a CoImmunoprecipitation Kit (Thermo Scientific), in accordance with manufacturer's protocol. Briefly, the purified monoclonal antibody was immobilized on beads in a column, and then, the extracted total protein was incubated with the beads at $4{ }^{\circ} \mathrm{C}$ for $2 \mathrm{~h}$. After washing, the immunoprecipitate was eluted with acidic buffer ( $\mathrm{pH}$ 2.8). The eluted fraction containing the antigen was denatured, separated by SDS-PAGE under reducing conditions, and stained with silver. The protein band indicating the antigen identified by matching with the Western blot was excised and cut into pieces. The excised gel piece was digested with modified porcine trypsin (Thermo Scientific), and the processed peptides were subjected to LC/MS/MS analysis with a Zaprous LC/MS system (AMR) at the National Institute for Materials Science (Tsukuba, Japan). The obtained data file was searched against the SWISSProt database using MASCOT MS/MS Ion Search (Matrix Science, London, UK).

\section{Immunohistochemistry for DDAH2 using hybridoma supernatant}

All samples were fixed with $10 \%$ formalin and embedded in paraffin. After deparaffinization, the samples were placed in blocking solution to suppress nonspecific staining for $30 \mathrm{~min}$. Then, antigen retrieval was performed using citrate buffer $(\mathrm{pH}$ 6.0) in an autoclave at $121^{\circ} \mathrm{C}$ for $15 \mathrm{~min}$. The culture supernatant of the hybridoma that detected DDAH2 was used as the primary antibody, and incubation was for $60 \mathrm{~min}$ at room temperature, followed by incubation with the secondary antibody (EnVision kit; Dako, Tokyo, Japan) for $30 \mathrm{~min}$ at room temperature. Diaminobenzidine chromogen solution (Dako) was applied to the slides, followed by counterstaining with hematoxylin. Immunohistochemistry for endothelial nitric oxide synthase (eNOS) and CD31 were performed in the same way, using anti-eNOS antibody at dilution of 1:75 (ab5589, Abcam, Cambridge, UK), and anti-CD31 antibody at dilution of 1:125 (ab28364, Abcam), respectively.

The staining was judged by focusing on the tumor stroma, and normal vascular endothelium was used as a control. If an area, showing staining similar to or stronger than that in the control, exceeded $5 \%$ of the total tumor stroma, we defined the staining as "positive." We further divided positive samples into two groups as follows: "DDAH2-strong" with a positive area stronger than the control evident in $30 \%$ or more of the stroma, and "DDAH2-weak" with a positive area of less than $30 \%$.

\section{DDAH2 in situ hybridization}

To identify cells that secreted DDAH2, in situ hybridization was performed as reported previously [13]. The details of in situ hybridization are described in the supplementary information.

\section{Western blot analysis of DDAH2 and eNOS}

Protein extraction from surgically resected specimens was performed using T-PER reagent (Pierce, Rockford, IL). Western blotting for DDAH2 was performed using the same protocol as that described above. Western blotting for eNOS was performed as follows. Twenty micrograms of extracted protein was mixed with Laemmli sample buffer, denatured at $95{ }^{\circ} \mathrm{C}$ for $5 \mathrm{~min}$, and electrophoresed on 7.5\% Mini Protein TGX gel (Bio-Rad Laboratories, Hercules, CA). Proteins were transferred to polyvinylidene difluoride (PVDF) membranes using an iBlot ${ }^{\mathrm{TM}}$ gel transfer system (Life Technologies Japan). The membranes were blocked with $0.1 \%$ blocking reagent and then incubated with anti-eNOS antibody at dilution of 1:1000 (ab5589, Abcam) at $4{ }^{\circ} \mathrm{C}$ overnight. After washing, immunoreactivity was detected with specific secondary antibodies conjugated to horseradish peroxidase. The protein bands were visualized using Supersignal West Femto maximum sensitivity substrate (Thermo Scientific) and X-ray film (BioMax Light film; Kodak, Rochester, NY).

\section{Cell culture and reagents}

Human umbilical vein endothelial cells (HUVECs) were cultured to evaluate the effect of DDAH2 on angiogenesis in vitro. The details are described in the supplementary information.

\section{Proliferation and capillary-like tube formation assay}

The details of endothelial cell proliferation and capillary-like tube formation assay are described in the supplementary information.

\section{Capillary-like tube formation assay}

To evaluate migration of endothelial cells, we examined capillary-like tube formation by the Geltre ${ }^{\mathrm{TM}}$ angiogenesis assay in accordance with manufacturer's protocol. Briefly, Geltrex ${ }^{\mathrm{TM}}$ was added to the growth surface and incubated for $30 \mathrm{~min}$ at $37{ }^{\circ} \mathrm{C}$ to allow the gel to solidify. HUVEC were gently added to each Geltrex ${ }^{\mathrm{TM}}$ coated 24 -well plate at $5 \times 10^{4}$ 
cells per well in growth medium. Then, DDAH2 recombinant protein or PBS (as control) was added. HUVEC were incubated for $6 \mathrm{~h}$ at $37{ }^{\circ} \mathrm{C}$ in an atmosphere containing $5 \% \mathrm{CO}_{2}$. The cells were visualized directly using a light microscope, and the mean tube length was measured.

\section{Statistical analysis}

Statistical analysis was performed using JMP 9 software. Statistical comparisons between groups were performed using one-way analysis of variance (ANOVA) followed by Student's $t$ test. Disease-free survival (DFS) with DDAH2 expression was compared using the Kaplan-Meier method, and the significance of differences between survival curves was assessed using log-rank test. DFS was determined from the date of surgery until the date of recurrence or last follow-up. Statistical significance was defined as $p<0.05$.

\section{Results}

\section{Screening for mouse monoclonal antibodies}

Ninety-seven hybridoma clones were obtained, and the hybridoma supernatants were used for immunohistochemical screening in two steps. As the first screening, we selected clones that were reactive with fetal but not with mature swine lung (Fig. 1a, b) and obtained 39 clones. We then selected antibodies reactive with lung adenocarcinoma but nonreactive with normal lung tissue, and obtained 11 clones. Of these clones, six showed cytoplasmic staining of cancer cells, but not with normal lung tissue. Four clones showed cytoplasmic staining of cancer cells but also nuclear staining of alveolar type II cells in normal lung tissue. We then immunohistochemically tested these clones on more than 100 adenocarcinomas and found that specificity for lung adenocarcinoma was low. One clone showed specific reactivity with cancer stroma but not with cancer cells (Fig. 1c, d). Because this pattern of reactivity was very characteristic, we selected this clone for further analysis.

\section{Identification of dimethylarginine dimethylaminohydrolase 2}

Using Western blotting, the clone was not reactive with a lung adenocarcinoma cell line (Calu-3) but the sample of lung adenocarcinoma showed a positive band at approximately $30 \mathrm{kDa}$ (Fig. 1e). By immunoprecipitation, we then isolated the recognized antigen in frozen human lung adenocarcinoma tissue, and identified this using LC-MS/MS as DDAH2.

\section{DDAH2 expression in lung adenocarcinoma}

DDAH2 diffusely stained cancer stroma, but the expressing cells could not be identified. Using in situ hybridization, we identified the cells expressing DDAH2 as fibroblasts in the malignant stroma (Fig. 2a-c). Using immunohistochemistry, we then examined expression of DDAH2 on 133 cases of surgically resected lung adenocarcinoma. The stroma of lung adenocarcinoma showed diffuse staining for DDAH2, whereas tumor cells themselves were not stained (Fig. $2 \mathrm{~d}-\mathrm{f}$ ). On the other hand, in normal lung tissue, only vascular endothelium showed staining for DDAH2. Table 1 summarizes the proportion of DDAH2-positive cases for each histological subtype of lung cancer. Interestingly, almost all cases of MIA and invasive adenocarcinoma were positive for DDAH2 (MIA $100 \%$; invasive adenocarcinoma $99 \%$ ), while only half of pre-invasive lesions were positive (AAH and AIS $46 \%$ ). The staining intensity was not clearly associated with histological evidence of invasion in the pleura or in vessels, or proliferating fibroblasts.

\section{DDAH2 expression and patient outcome}

We assessed association of DDAH2 expression with patient outcome. We selected 61 pathological stage I cases of which details of the postoperative course were available. The samples were divided into a DDAH2-strong group (Fig. 3a, $n=26$ ) and a DDAH2-weak group (Fig. 3b, $n=35$ ). The KaplanMeier curves showed a significant difference in DFS between the DDAH2-strong and DDAH2-weak groups $(p=0.026$, Fig. 3c). High expression of DDAH2 was significantly associated with poor outcome.

\section{DDAH2 and tumor angiogenesis}

We next examined whether DDAH2 might be a prognostic factor for lung adenocarcinoma. We hypothesized that DDAH2 contributes to lung adenocarcinoma invasion through tumor angiogenesis via NO production (Fig. 4a) and expected that eNOS expression might increase upon stimulation by DDAH2. Expression of both DDAH2 and eNOS by Western blotting on seven surgically resected specimens, showed both to be expressed at a significantly higher level in invasive adenocarcinoma than in AIS and normal lung (Fig. 4b). By IHC, we confirmed high expression of eNOS in invasive adenocarcinoma compared to AIS and normal lung, using ten invasive adenocarcinomas, five AIS, and five normal lung tissues. Interestingly, eNOS was expressed strongly in vascular endothelium of malignant stroma (Fig. 4c, d), whereas vascular endothelium of normal lung was negative or showed only faint staining (Fig. 4e, f). These data indicate that DDAH2 stimulated production of eNOS is significantly higher in invasive adenocarcinoma than in AIS and normal tissue. 

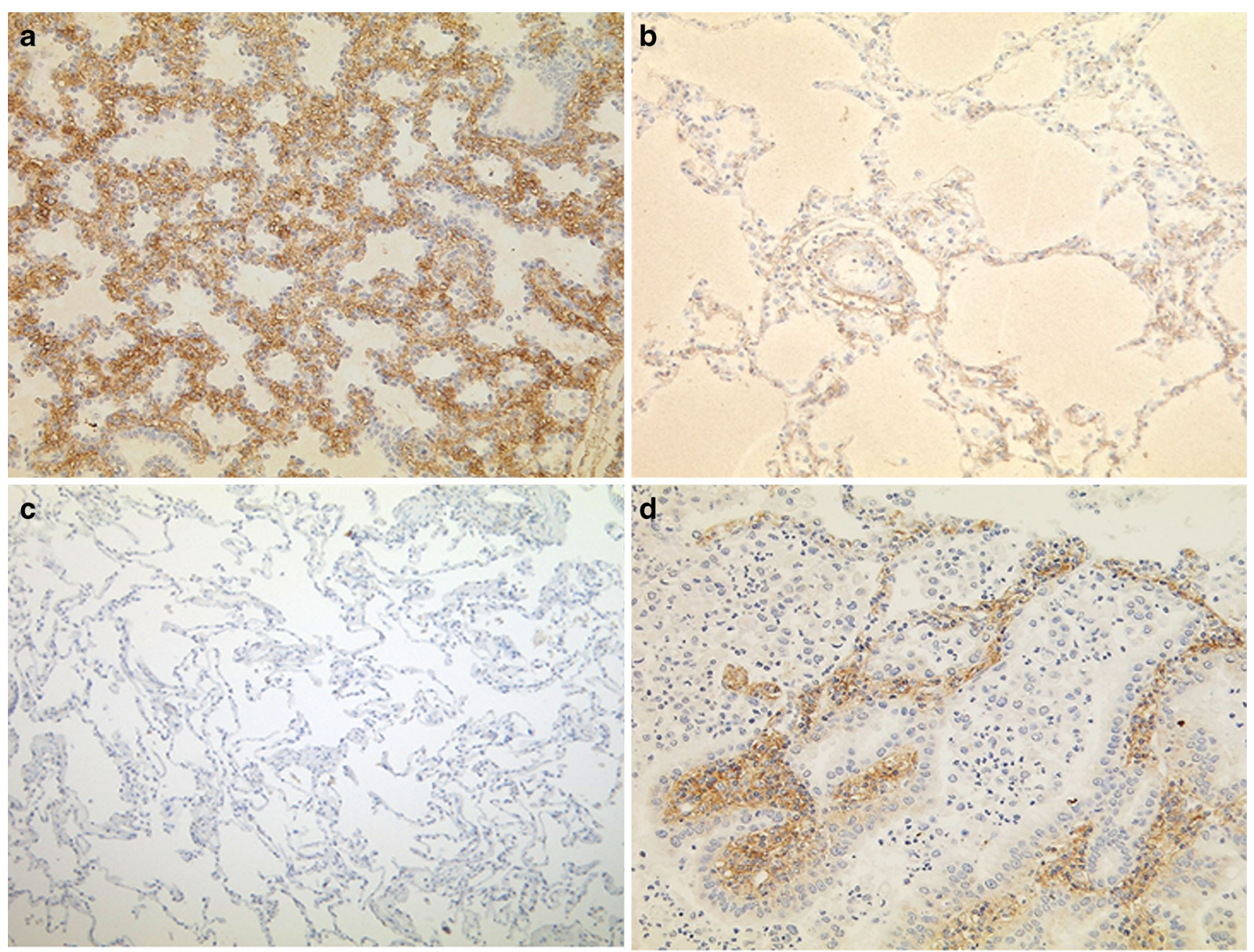

e

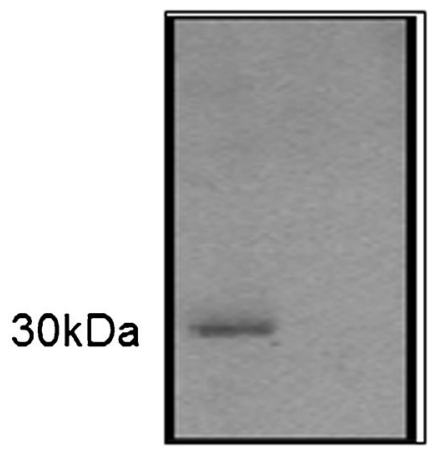

\section{(1) (2)}

Fig. 1 Immunohistochemical screening using culture supernatant of hybridoma clones. Initial screening was performed using swine lung tissues, and we selected clones that positively recognized antigens in fetal miniature swine lung (a) and were unreactive with mature swine lung (b). In the second screening, normal lung tissue and small lung adenocarcinoma were used and the clones reactive only with lung

To determine whether DDAH2 regulates angiogenesis through NO production, we next examined the angiogenic effect of DDAH2 in vitro. We first confirmed by Western blotting that DDAH2 recombinant protein significantly

adenocarcinoma were selected. c Absence of staining in normal human lung. d Staining of tumor stroma of lung adenocarcinoma. e Western blotting using the selected hybridoma supernatant as the primary antibody. Protein bands were detected at approximately $30 \mathrm{kDa}$ with protein extracted from frozen lung adenocarcinoma specimens (1), but not from the lung cancer cell line, Calu-3 (2)

enhances eNOS expression in HUVEC (data not shown). We then found that recombinant DDAH2 protein significantly increases the number of HUVEC in a dose-dependent manner (Fig. 5a, c). We also examined the effect of DDAH2 on 

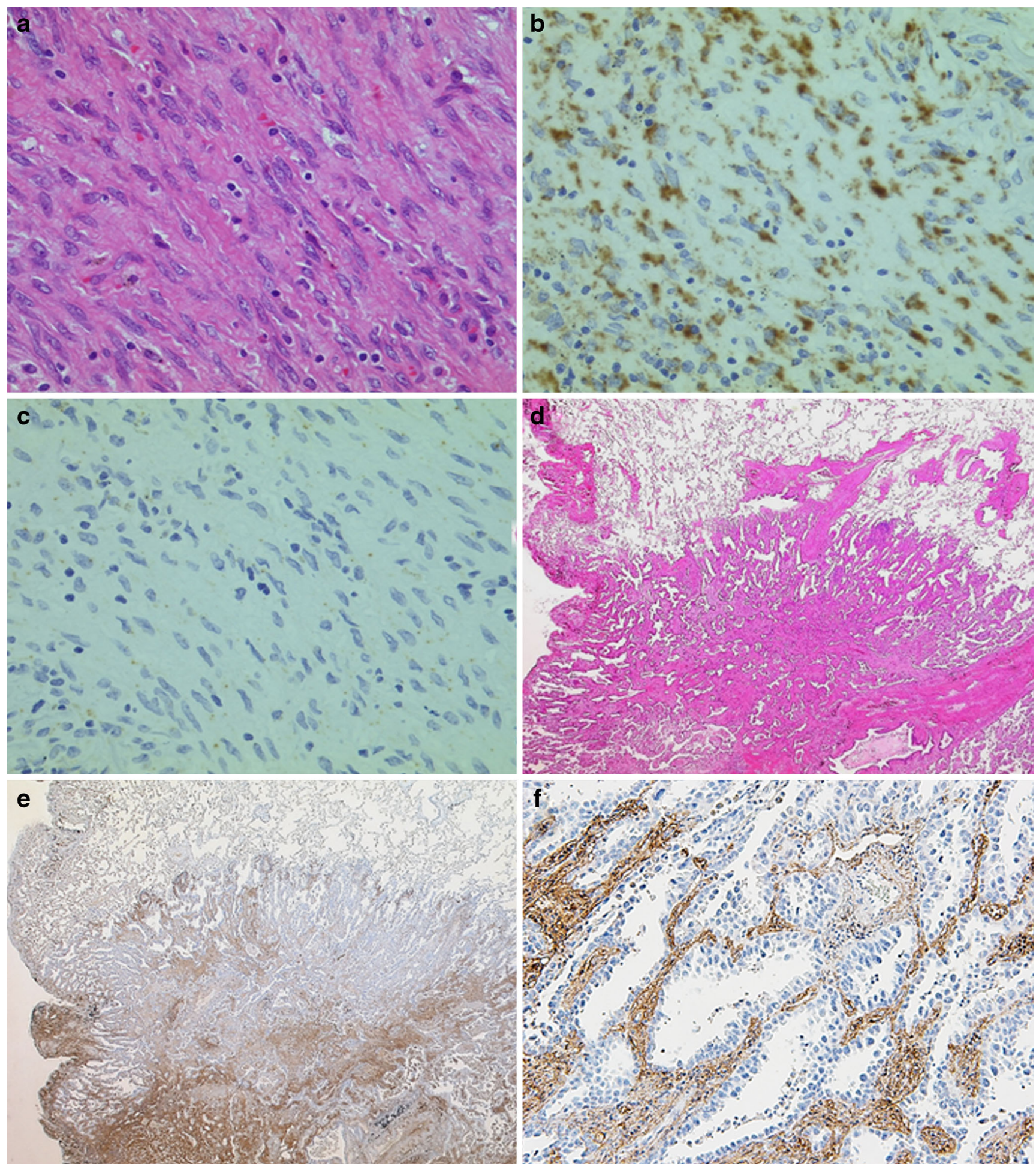

Fig. 2 In situ hybridization (ISH) of DDAH2 mRNA using lepidicpredominant invasive adenocarcinoma. a Fibroblasts in tumor stroma (HE stain). b Fibroblasts are reactive for DDAH2 mRNA with antisense cRNA probe. $\mathbf{c}$ No reactivity was found when a sense-cRNA probe of

DDAH2 mRNA was used. Immunohistochemistry for DDAH2 in lung adenocarcinoma. d Representative case of adenocarcinoma showing a lepidic growth pattern. e DDAH2-IHC $(\times 10)$ and $(\mathbf{f})$ DDAH2-IHC $(\times 40)$; the tumor stroma diffusely stains whereas tumor cells are negative

HUVEC tube formation in an in vitro angiogenesis model. As shown in Fig. 5b, d, DDAH2 recombinant protein significantly enhances capillary-like tube formation in comparison with

the control group. Taken together, our findings suggest that DDAH2 promotes tumor angiogenesis in lung adenocarcinoma by increasing the production of NO. 
Table 1 Proportion of positive cases for DDAH2 immunohistochemistry

\begin{tabular}{ll}
\hline Histological subtypes & Positive cases \\
\hline Preinvasive lesion & $21 / 47(46 \%)$ \\
Atypical adenomatous hyperplasia (AAH) & $2 / 14$ \\
Adenocarcinoma in situ (AIS) & $19 / 33$ \\
Minimally invasive adenocarcinoma (MIA) & $11 / 11(100 \%)$ \\
Invasive adenocarcinoma & $74 / 75(99 \%)$ \\
Lepidic predominant & $40 / 41$ \\
Acinar predominant & $7 / 7$ \\
Papillary predominant & $8 / 8$ \\
Micropapillary predominant & $1 / 1$ \\
Solid predominant & $18 / 18$ \\
\hline
\end{tabular}
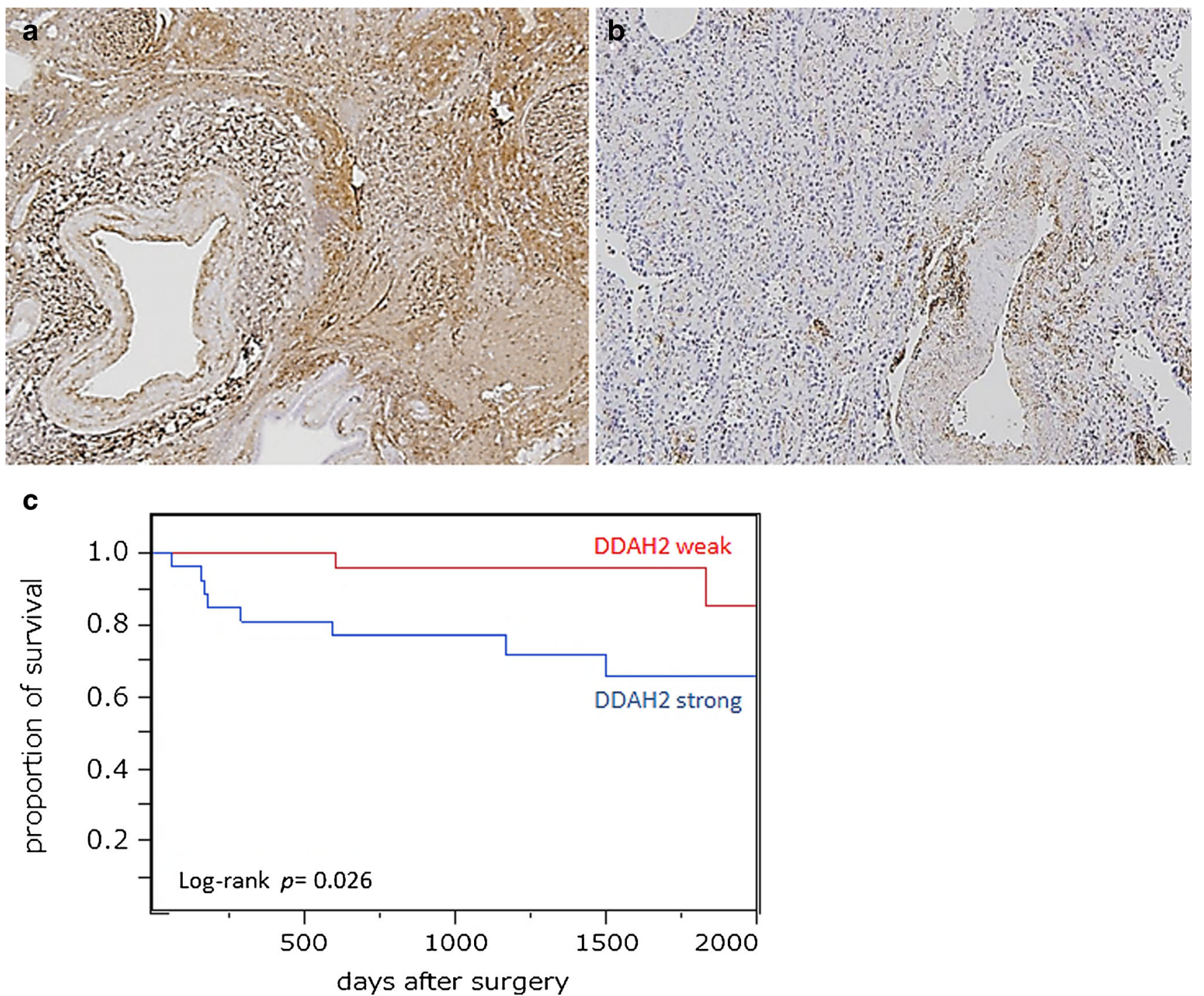

Fig. 3 Based on intensity of immunohistochemical staining, 61 cases of stage I adenocarcinoma were divided into (a) DDAH2-strong and (b) DDAH2weak. c Kaplan-Meier analysis of disease-free survival using log-rank test 


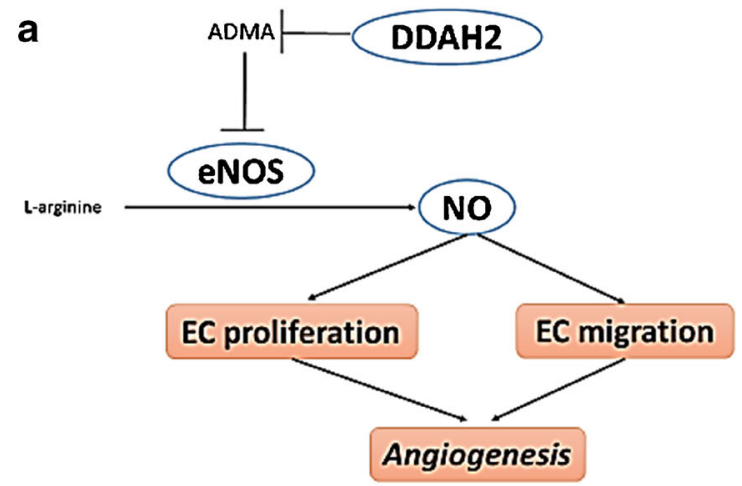

b

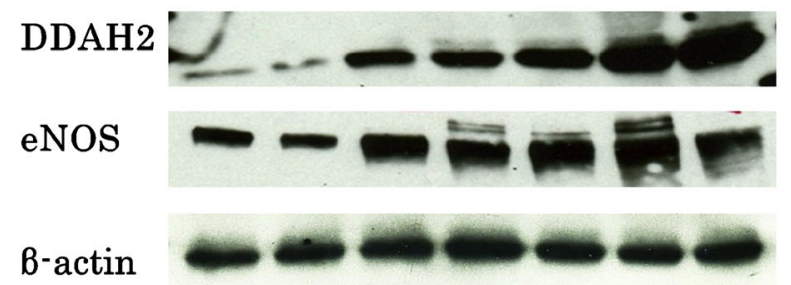

Normal AIS Invasive adenocarcinoma $(\mathrm{n}=1) \quad(\mathrm{n}=1) \quad(\mathrm{n}=5)$

EC: EndothelialCell

\section{C}

\section{d}
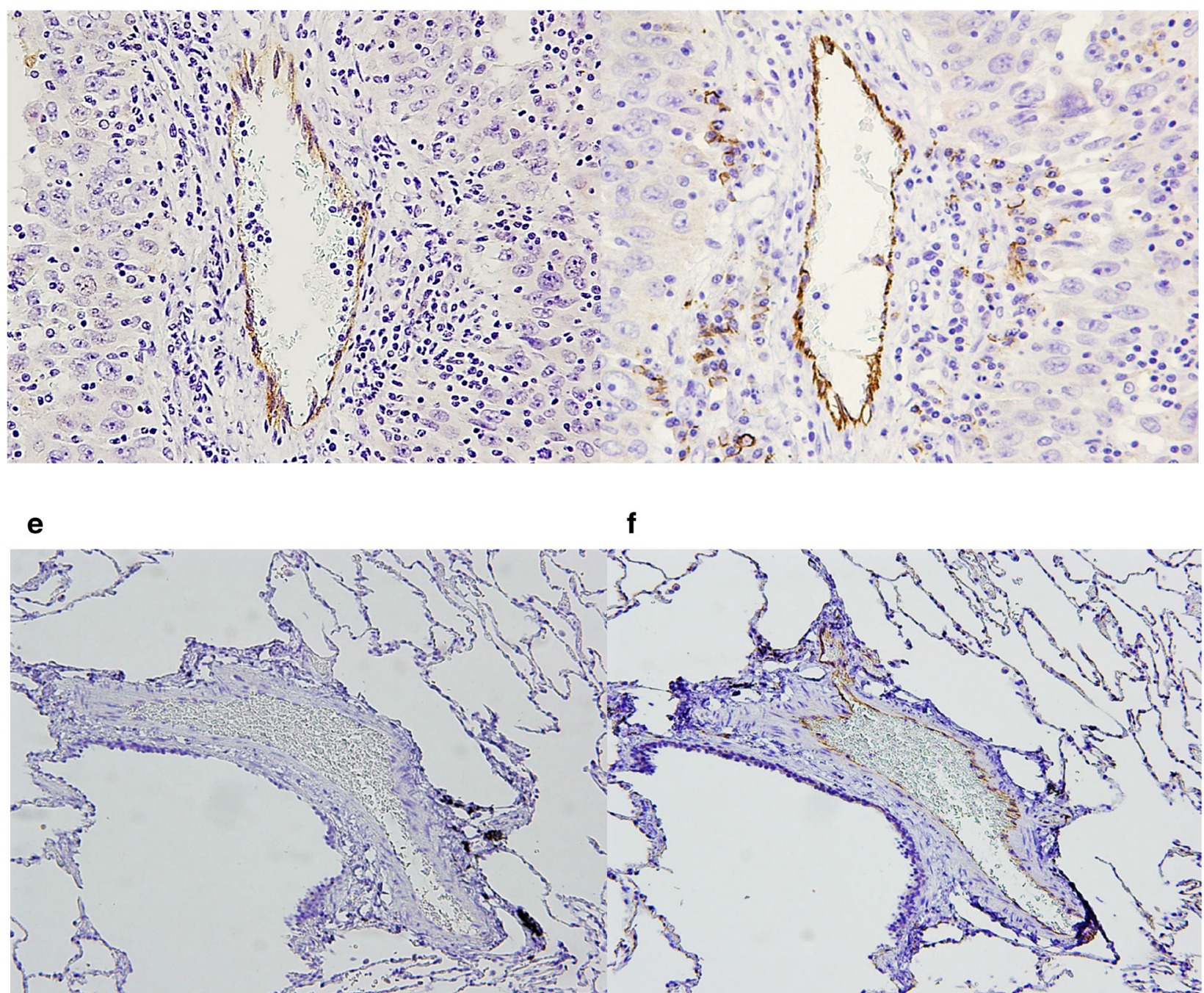

Fig. 4 DDAH2 promotes tumor angiogenesis through NO production in lung adenocarcinoma. a Tumor fibroblast-derived DDAH2 increases expression of eNOS in vascular endothelial cells, and enhances NO production, followed by upregulation of the kinase cascade. This pathway stimulates endothelial cell proliferation and migration and results in angiogenesis. b Samples of normal lung $(n=1)$, AIS $(n=1)$, and invasive adenocarcinoma $(n=5)$ were prepared and used for Western blot analysis with antibodies against DDAH2 and eNOS.
Immunohistochemistry with anti-eNOS antibody was performed using the same samples as those used for Western blotting. Vascular endothelial cells were subjected to immunohistochemistry with antiCD31 antibody. c eNOS was expressed strongly in vascular endothelium of the invasive adenocarcinoma. d Immunohistochemistry with anti-CD31 antibody in the same section as c. e The vascular endothelial cells of normal lung were negative for eNOS. f Immunohistochemistry with anti-CD31 antibody in the same section as $\mathbf{e}$ 
proteins highly expressed in several fetal tissues, including lung and kidney. In hypoplastic human fetal lung, vascular endothelium showed significantly decreased expression of both CD31 and eNOS relative to normal control tissue [17]. It has been suggested that the DDAH2-eNOS pathway plays an important role in the process of angiogenesis in fetal lung. On the other hand, DDAH2 is also regarded as an antiatherosclerotic factor in adults because DDAH2 increases the production of NO, which promotes vasodilation and angiogenesis. Although impairment or decreased expression of DDAH2 has been reported in several diseases such as chronic kidney disease, pulmonary hypertension, and cardiovascular disease [18-22], the association of DDAH2 with malignancy has received less attention.

We initially examined expression of DDAH2 in lung adenocarcinomas using immunohistochemistry and found a significant difference in the proportion of DDAH2-positive cases between MIA, invasive adenocarcinoma, and preinvasive lesions. DDAH2 was expressed in most invasive adenocarcinomas, but only about half of the AIS cases showed DDAH2 staining. Because most lung adenocarcinomas develop in a stepwise manner from AAH to AIS, and then from MIA to invasive adenocarcinoma, it has been suggested that DDAH2 might play an important role in the progression of lung adenocarcinoma, especially in the early phases of tumor invasion. Moreover, we found a statistically significant correlation between the intensity of immunohistochemical staining for DDAH2 and DFS. This suggests that DDAH2 might be a novel prognostic indicator in lung adenocarcinoma and useful for the detection of early invasive adenocarcinoma such as MIA.

We hypothesized that DDAH2 contributes to lung adenocarcinoma invasion through promotion of tumor angiogenesis. As shown in Fig. 4a, expression of tumor fibroblast-derived DDAH2 in vascular endothelium led to an increase of NO production, followed by increased vascular endothelial cell proliferation and migration. Angiogenesis is an important process for tumor growth, and several therapeutic strategies targeting tumor angiogenesis have been developed [23-25]. Only a few previous studies have investigated the function of DDAH in malignant tumors. Kostourou et al. reported that overexpression of DDAH1 is associated with increased neovascularization of gliomas in vivo, and Vanella et al. suggested that DDAH2 might contribute to tumor angiogenesis in prostate cancer through an increase of NO production [26, 27]. Both studies suggest an angiogenic effect of DDAH in malignant tumors. Against this background, we initially examined expression of DDAH2 and eNOS in surgical specimens. eNOS was more highly expressed in invasive adenocarcinoma than in normal tissue or AIS, reflecting the pattern of DDAH2 expression. We then showed that DDAH2 significantly promotes proliferation and capillary-like tube formation of endothelial cells in vitro. These results suggest that tumor angiogenesis in invasive adenocarcinoma is at least partially activated by DDAH2. It is of considerable interest that DDAH2 accelerates the progression of lung adenocarcinoma whereas it works as a preventive factor against cardiovascular and chronic kidney diseases.

One of the noteworthy findings of this study was that DDAH2 is expressed in cancer-associated fibroblasts (CAF) of lung adenocarcinoma. CAF are activated fibroblasts observed in the stroma of various solid tumors. CAF are a major constituent of reactive tumor stroma and play a crucial role in tumor progression and metastasis $[28,29]$. Recently, emerging evidence has suggested that $\mathrm{CAF}$ also promote tumor angiogenesis in breast and colon cancer by secreting angiogenic factors [30, 31]. In lung adenocarcinoma, previous reports have indicated that CAF play an important role in cancer progression and that their presence is correlated with poor prognosis $[32,33]$. However, a role for $\mathrm{CAF}$ in angiogenesis in lung adenocarcinoma has not been well characterized. We observed diffuse staining of DDAH2 in cancer stroma and detected expression of DDAH2 mRNA in CAF of lung adenocarcinoma by in situ hybridization. We also found that DDAH2 derived from CAF promotes tumor angiogenesis in lung adenocarcinoma. These results suggest that $\mathrm{CAF}$ contribute to tumor angiogenesis in lung adenocarcinoma by secreting DDAH2.

In lung cancer, the angiogenic vascular endothelial growth factor (VEGF) is expressed mainly in tumor cells, and bevacizumab, a humanized anti-VEGF monoclonal antibody, has been developed and is applied as systemic therapy in patients with nonsmall cell lung cancer, with the exception of squamous cell carcinoma [34-36]. However, for control of tumor angiogenesis in lung cancer inhibition of VEGF only is insufficient. Possible functional relationships between VEGF and DDAH2 in lung adenocarcinoma need to be studied. During tumor angiogenesis, VEGF stimulates NO production via activation of the PI3K/Akt cascade, which is distinct from the DDAH/ADMA pathway $[37,38]$. Several reports have described a role of DDAH2 in regulating VEGF expression. Hasegawa et al. reported that DDAH2 stimulates expression of VEGF through the transcription factor $\mathrm{Sp} 1$ in vascular endothelial cells, and Xiao et al. reported that expression of VEGF mRNA in endothelial cells is significantly suppressed by DDAH2 siRNA [39, 40]. These data suggest that DDAH2 exerts dual effects on NOmediated angiogenesis. DDAH2 may act not only on vascular endothelial cells by releasing them from ADMA-induced eNOS inhibition but also on tumor cells by stimulating their expression of VEGF. Inhibition of DDAH2 activity in lung adenocarcinoma may therefore become a promising therapeutic strategy complementing conventional antiangiogenic therapy.

We show that DDAH2 plays an important role in tumor angiogenesis in lung adenocarcinoma. However, the mechanism by which DDAH2 expression is regulated in malignant stroma remains unclear. Malignant stroma is an aberrant microenvironment, characterized by persistent hypoxic conditions. Hypoxia is one of the major features of solid tumors, favoring tumor progression and increasing the degree of tumor resistance to treatment $[41,42]$. Emerging evidence suggests that hypoxia- 
a

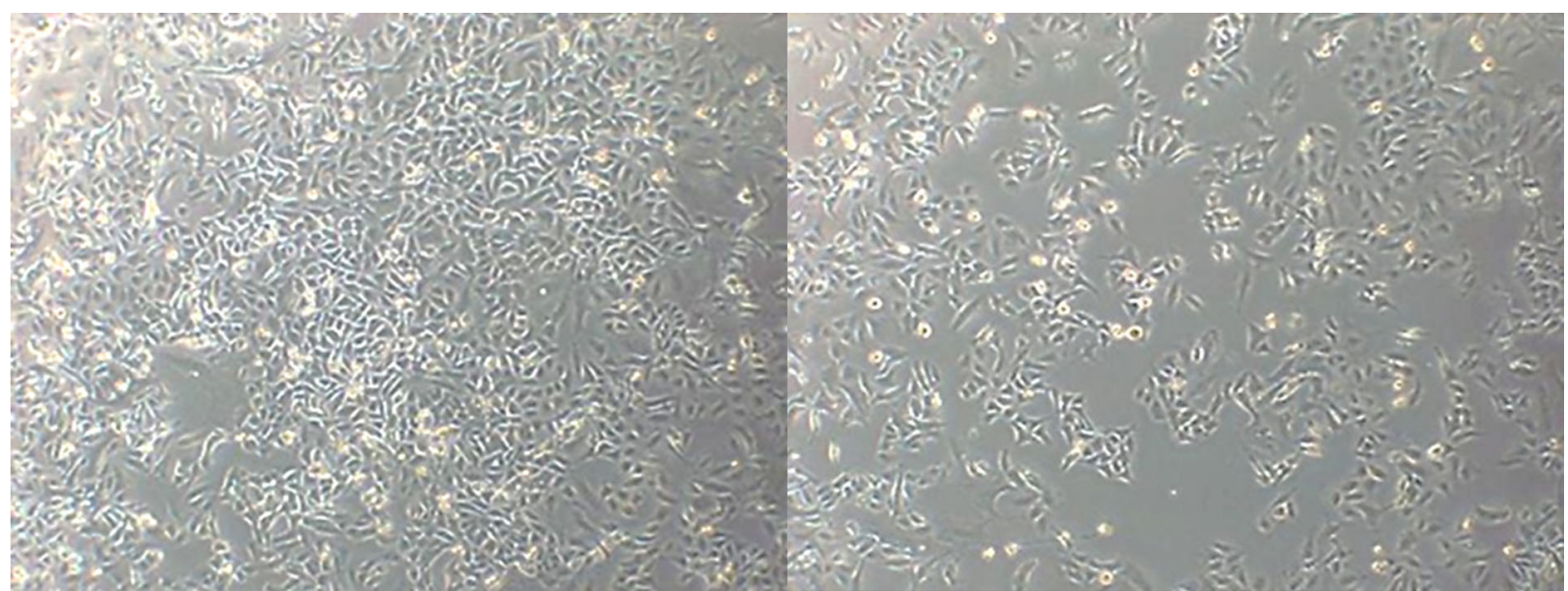

DDAH2 $(0.50 \mu \mathrm{g} / \mathrm{ml})$

\section{PBS}

b

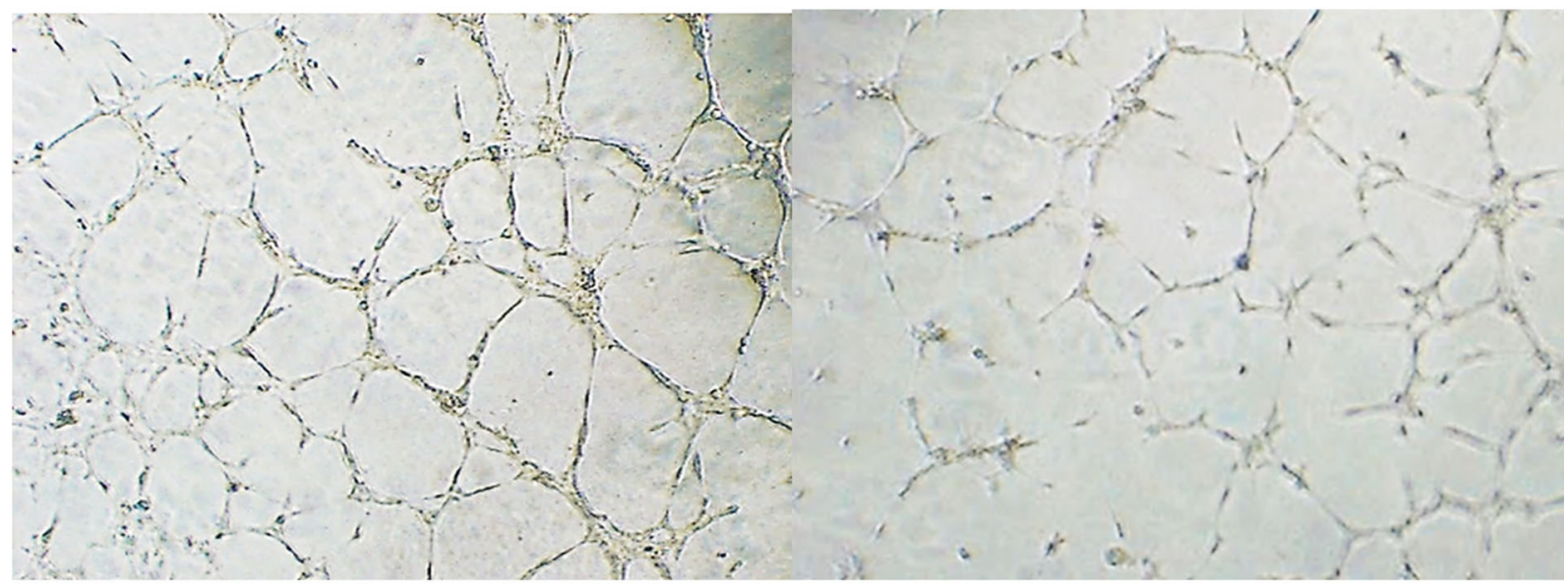

\section{DDAH2 $(0.50 \mu \mathrm{g} / \mathrm{ml})$}

\section{PBS}
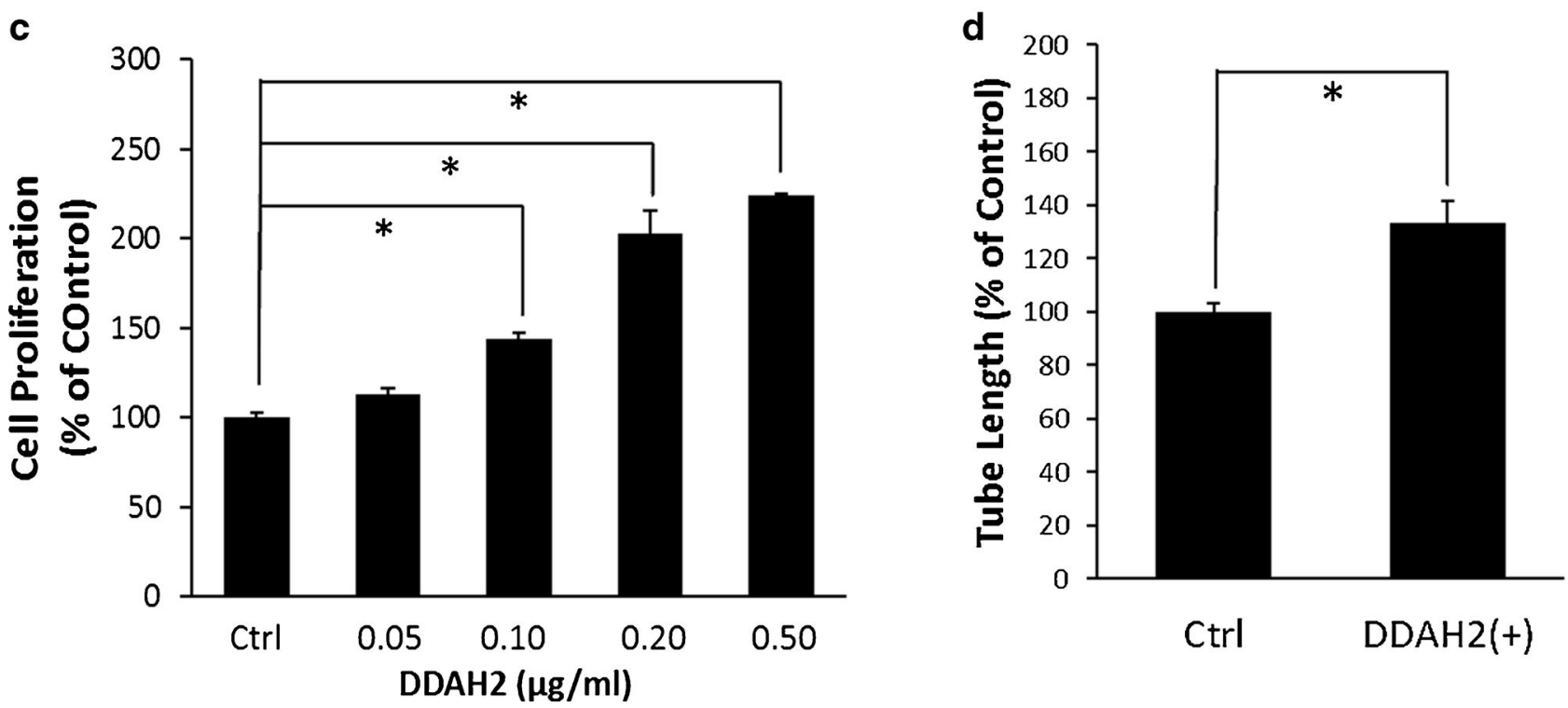
4 Fig. 5 DDAH2 induces proliferation of, and capillary-like tube formation by HUVEC. a Recombinant human DDAH2 protein was added to HUVEC at various doses $(0.05-0.5 \mu \mathrm{g} / \mathrm{ml})$ for $48 \mathrm{~h}$. PBS was used as control. b Representative photographs illustrate the effects of recombinant human DDAH2 protein on capillary-like tube formation by HUVEC. $\mathbf{c}$ Cell proliferation was determined by direct counting. $\mathbf{d}$ The mean tube length in each well was measured. These experiments were performed in duplicate. ${ }^{*} p<0.05$

inducible signaling pathways are significantly associated with tumor angiogenesis [43, 44]. Among the major transcription regulators involved in the response to hypoxic conditions, hypoxia-inducible factor (HIF) plays an important role in tumor angiogenesis, invasion, and metastasis. HIF promotes tumor angiogenesis through activation of downstream genes, such as VEGF and angiopoetin-2 (Ang-2) [45, 46]. Although the association between hypoxia and DDAH2 is still unclear, DDAH2 might be regulated by HIF, as well as other angiogenic factors. Pullamsetti et al. reported that in murine pulmonary fibrosis transforming growth factor- $\beta 1$ (TGF- $\beta 1$ ) expressed by lung epithelial cells increases the expression of DDAH2 in a timedependent manner [47]. TGF- $\beta 1$ is considered to be one of the key regulators of tumor progression, notably in the process of epithelial-mesenchymal transition (EMT) [48]. Several reports have suggested that TGF- $\beta 1$ also accelerates tumor angiogenesis $[49,50]$. These molecules might be potential regulators of DDAH2 in malignant stroma, and further studies aimed at clarifying the mechanism of DDAH2 regulation are needed.

In conclusion, DDAH2 is expressed in CAF in early stage lung adenocarcinoma and might play an important role in tumor invasion by promoting tumor angiogenesis through an increase of NO production. It also might be a novel prognostic factor in lung adenocarcinoma. To elucidate the mechanisms involved in vivo studies will be necessary. The therapeutic potential of DDAH2 as target for inhibition of tumor angiogenesis in lung adenocarcinoma, complementary to conventional antiangiogenic therapy, merits to be explored.

\begin{abstract}
Acknowledgements This study was supported by NIMS Molecule and Material Synthesis Platform in "Nanotechnology Platform Project" operated by the Ministry of Education, Culture, Sports, Science and Technology (MEXT), Japan. This study was also supported in part by Grants-inAid for Scientific Research from the Ministry of Education, Culture, Sports, Science and Technology of Japan (no. 26670172) to M.N. No additional external funding received for this study.
\end{abstract}

Conflict of interest The authors declare that they have no conflict of interest.

Open Access This article is distributed under the terms of the Creative Commons Attribution 4.0 International License (http:// creativecommons.org/licenses/by/4.0/), which permits unrestricted use, distribution, and reproduction in any medium, provided you give appropriate credit to the original author(s) and the source, provide a link to the Creative Commons license, and indicate if changes were made.

\section{References}

1. Travis WD, Lubin J, Ries L, Devesa S (1996) United States lung carcinoma incidence trends: declining for most histologic types among males, increasing among females. Cancer 77:2464-2470

2. Valaitis J, Warren S, Gamble D (1981) Increasing incidence of adenocarcinoma of the lung. Cancer 47:1042-1046

3. Travis WD, Brambilla E, Noguchi M, Nicholson AG, Geisinger KR, Yatabe Y, Beer DG, Powell CA, Riely GJ, Van Schil PE, Garg K, Austin JH, Asamura H, Rusch VW, Hirsch FR, Scagliotti G, Mitsudomi T, Huber RM, Ishikawa Y, Jett J, Sanchez-Cespedes M, Sculier JP, Takahashi T, Tsuboi M, Vansteenkiste J, Wistuba I, Yang PC, Aberle D, Brambilla C, Flieder D, Franklin W, Gazdar A, Gould M, Hasleton P, Henderson D, Johnson B, Johnson D, Kerr K, Kuriyama K, Lee JS, Miller VA, Petersen I, Roggli V, Rosell R, Saijo N, Thunnissen E, Tsao M, Yankelewitz D (2011) International Association for the Study of Lung Cancer/American Thoracic Society/European Respiratory Society: international multidisciplinary classification of lung adenocarcinoma. J Thorac Oncol 6:244-285

4. Travis WD, Brambilla E, Riely GJ (2013) New pathologic classification of lung cancer: relevance for clinical practice and clinical trials. J Clin Oncol 31:992-1001

5. Noguchi M (2010) Stepwise progression of pulmonary adenocarcinoma-clinical and molecular implications. Cancer Metastasis Rev 29:15-21

6. Cardarella S, Johnson BE (2013) The impact of genomic changes on treatment of lung cancer. Am J Respir Crit Care Med 188: 770-775

7. Gainor JF, Shaw AT (2013) Novel targets in non-small cell lung cancer: ROS1 and RET fusions. Oncologist 18:865-875

8. Mazières J, Peters S, Lepage B, Cortot AB, Barlesi F, Beau-Faller M (2013) Lung cancer that harbors an HER2 mutation: epidemiologic characteristics and therapeutic perspectives. J Clin Oncol 31: 1997-2003

9. Grunnet M, Sorensen JB (2012) Carcinoembryonic antigen (CEA) as tumor marker in lung cancer. Lung Cancer 76:138-143

10. De Jong J, Looijenga LH (2006) Stem cell marker OCT3/4 in tumor biology and germ cell tumor diagnostics: history and future. Crit Rev Oncog 12:171-203

11. Mimeault M, Batra SK (2014) Molecular biomarkers of cancer stem/progenitor cells associated with progression, metastases, and treatment resistance of aggressive cancers. Cancer Epidemiol Biomarkers Prev 23:234-254

12. Sobin LK, Gospodarowicz M, Wittekind C (2009) International Union Against Cancer: TNM classification of Malignant tumors, 7th edn. Wiley-Blackwell, West Sussex, pp 136-146

13. Pei Y, Kano J, Iijima T, Morishita Y, Inadome Y, Noguchi M (2009) Overexpression of Dickkopf 3 in hepatoblastomas and hepatocellular carcinomas. Virchows Arch 454:639-646

14. Tran CT, Fox MF, Vallance P, Leiper JM (2000) Chromosomal localization, gene structure, and expression pattern of DDAH1: comparison with DDAH2 and implications for evolutionary origins. Genomics 68:101-105

15. Fiedler LR, Wojciak-Stothard B (2009) The DDAH/ADMA pathway in the control of endothelial cell migration and angiogenesis. Biochem Soc Trans 37:1243-1247

16. Palm F, Onozato ML, Luo Z, Wilcox CS (2007) Dimethylarginine dimethylaminohydrolase (DDAH): expression, regulation, and function in the cardiovascular and renal systems. Am J Physiol Heart Circ Physiol 293:3227-3245

17. Boucherat O, Franco-Montoya ML, Delacourt C, Martinovic J, Masse V, Elie C, Thébaud B, Benachi A, Bourbon JR (2010) Defective angiogenesis in hypoplastic human fetal lungs correlates with nitric oxide synthase deficiency that occurs despite enhanced 
angiopoietin-2 and VEGF. Am J Physiol Lung Cell Mol Physiol 298:849-856

18. Leiper J, Nandi M (2011) The therapeutic potential of targeting endogenous inhibitors of nitric oxide synthesis. Nat Rev Drug Discov 10:277-291

19. Matsuguma K, Ueda S, Yamagishi S, Matsumoto Y, Kaneyuki U, Shibata R, Fujimura T, Matsuoka H, Kimoto M, Kato S, Imaizumi T, Okuda S (2006) Molecular mechanism for elevation of asymmetric dimethylarginine and its role for hypertension in chronic kidney disease. J Am Soc Nephrol 17:2176-2183

20. Tatematsu S, Wakino S, Kanda T, Homma K, Yoshioka K, Hasegawa K, Sugano N, Kimoto M, Saruta T, Hayashi K (2007) Role of nitric oxide-producing and -degrading pathways in coronary endothelial dysfunction in chronic kidney disease. J Am Soc Nephrol 18:741-749

21. Arrigoni FI, Vallance P, Haworth SG, Leiper JM (2003) Metabolism of asymmetric dimethylarginines is regulated in the lung developmentally and with pulmonary hypertension induced by hypobaric hypoxia. Circulation 107:1195-1201

22. Pullamsetti S, Kiss L, Ghofrani HA, Voswinckel R, Haredza P, Klepetko W, Aigner C, Fink L, Muyal JP, Weissmann N, Grimminger F, Seeger W, Schermuly RT (2005) Increased levels and reduced catabolism of asymmetric and symmetric dimethylarginines in pulmonary hypertension. FASEB J 19: 1175-1177

23. Cao Y (2005) Tumor angiogenesis and therapy. Biomed Pharmacother 59(Suppl 2):340-343

24. Kirstein MN, Moore MM, Dudek AZ (2006) Review of selected patents for cancer therapy targeting tumor angiogenesis. Recent Pat Anticancer Drug Discov 1:153-161

25. Wachsberger P, Burd R, Dicker AP (2003) Tumor response to ionizing radiation combined with antiangiogenesis or vascular targeting agents: exploring mechanisms of interaction. Clin Cancer Res 9:1957-1971

26. Kostourou V, Robinson SP, Whitley GS, Griffiths JR (2003) Effects of overexpression of dimethylarginine dimethylaminohydrolase on tumor angiogenesis assessed by susceptibility magnetic resonance imaging. Cancer Res 63:4960-4966

27. Vanella L, Di Giacomo C, Acquaviva R, Santangelo R, Cardile V, Barbagallo I, Abraham NG, Sorrenti V (2011) The DDAH/NOS pathway in human prostatic cancer cell lines: antiangiogenic effect of L-NAME. Int J Oncol 39:1303-1310

28. Franco OE, Shaw AK, Strand DW, Hayward SW (2010) Cancer associated fibroblasts in cancer pathogenesis. Semin Cell Dev Biol 21:33-39

29. Räsänen K, Vaheri A (2010) Activation of fibroblasts in cancer stroma. Exp Cell Res 316:2713-2722

30. Orimo A, Gupta PB, Sgroi DC, Arenzana-Seisdedos F, Delaunay T, Naeem R, Carey VJ, Richardson AL, Weinberg RA (2005) Stromal fibroblasts present in invasive human breast carcinomas promote tumor growth and angiogenesis through elevated SDF-1/CXCL12 secretion. Cell 121:335-348

31. Nagasaki T, Hara M, Nakanishi H, Takahashi H, Sato M, Takeyama H (2014) Interleukin-6 released by colon cancer-associated fibroblasts is critical for tumour angiogenesis: anti-interleukin-6 receptor antibody suppressed angiogenesis and inhibited tumour-stroma interaction. Br J Cancer 110:469-478

32. Kawase A, Ishii G, Nagai K, Ito T, Nagano T, Murata Y, Hishida T, Nishimura M, Yoshida J, Suzuki K, Ochiai A (2008) Podoplanin expression by cancer associated fibroblasts predicts poor prognosis of lung adenocarcinoma. Int J Cancer 123:1053-1059

33. Kinoshita T, Ishii G, Hiraoka N, Hirayama S, Yamauchi C, Aokage K, Hishida T, Yoshida J, Nagai K, Ochiai A (2013) Forkhead box
P3 regulatory T cells coexisting with cancer associated fibroblasts are correlated with a poor outcome in lung adenocarcinoma. Cancer Sci 104:409-415

34. Sandler A, Gray R, Perry MC, Brahmer J, Schiller JH, Dowlati A, Lilenbaum R, Johnson DH (2006) Paclitaxel-carboplatin alone or with bevacizumab for non-small-cell lung cancer. N Engl J Med 355:2542-2550

35. Reck M, von Pawel J, Zatloukal P, Ramlau R, Gorbounova V, Hirsh V, Leighl N, Mezger J, Archer V, Moore N, Manegold C (2009) Phase III trial of cisplatin plus gemcitabine with either placebo or bevacizumab as first-line therapy for nonsquamous non-small-cell lung cancer: AVAil. J Clin Oncol 27:1227-1234

36. Wagstaff AJ, Keam SJ, McCormack PL (2009) Bevacizumab plus platinum-based chemotherapy: in advanced non-small cell lung cancer. BioDrugs 23:187-196

37. Rebecca Y, Alex T (2009) Function of Akt/PKB Signaling to Cell Motility, Invasion and the Tumor Stroma in Cancer. Cell Signal 21: 470-476

38. Isenberg JS, Martin-Manso G, Maxhimer JB, Roberts DD (2009) Regulation of nitric oxide signaling by thrombospondin-1: implications for anti-angiogenic therapies. Nat Rev Cancer 9:182-194

39. Hasegawa K, Wakino S, Tanaka T, Kimoto M, Tatematsu S, Kanda T, Yoshioka K, Homma K, Sugano N, Kurabayashi M, Saruta T, Hayashi K (2006) Dimethylarginine dimethylaminohydrolase 2 increases vascular endothelial growth factor expression through $\mathrm{Sp} 1$ transcription factor in endothelial cells. Arterioscler Thromb Vasc Biol 26:1488-1494

40. Xiao J, Xiao ZJ, Liu ZG, Gong HY, Yuan Q, Wang S, Li YJ, Jiang DJ (2009) Involvement of dimethylarginine dimethylaminohydrolase-2 in visfatin-enhanced angiogenic function of endothelial cells. Diabetes Metab Res Rev 25:242-249

41. Noman MZ, Messai Y, Carré T, Akalay I, Méron M, Janji B, Hasmim M, Chouaib S (2011) Microenvironmental hypoxia orchestrating the cell stroma cross talk, tumor progression and antitumor response. Crit Rev Immunol 31:357-377

42. Lin Q, Yun Z (2010) Impact of the hypoxic tumor microenvironment on the regulation of cancer stem cell characteristics. Cancer Biol Ther 9:949-956

43. Moeller BJ, Cao Y, Vujaskovic Z, Li CY, Haroon ZA, Dewhirst MW (2004) The relationship between hypoxia and angiogenesis. Semin Radiat Oncol 14:215-221

44. Christopher P, Peter R (2003) Regulation of angiogenesis by hypoxia: role of the HIF system. Nat Med 9:677-684

45. Fujimoto J, Sato E, Alam SM, Jahan I, Toyoki H, Hong BL, Sakaguchi H, Tamaya T (2006) Plausible linkage of hypoxiainducible factor (HIF) in uterine endometrial cancers. Oncology 71:95-101

46. Lee OH, Xu J, Fueyo J, Alonso MM, Liu D, Martin V, Jiang H, Piao Y, Liu TJ, Gomez-Manzano C (2008) Angiopoietin-2 decreases vascular endothelial growth factor expression by modulating HIF1 alpha levels in gliomas. Oncogene 27:1310-1314

47. Pullamsetti SS, Savai R, Dumitrascu R, Dahal BK, Wilhelm J, Konigshoff M, Zakrzewicz D, Ghofrani HA, Weissmann N, Eickelberg O, Guenther A, Leiper J, Seeger W, Grimminger F, Schermuly RT (2011) The role of dimethylarginine dimethylaminohydrolase in idiopathic pulmonary fibrosis. Sci Transl Med 3:87ra53

48. Zavadil J, Böttinger EP (2005) TGF-beta and epithelial-tomesenchymal transitions. Oncogene 24:5764-5774

49. Jakowlew SB (2006) Transforming growth factor-beta in cancer and metastasis. Cancer Metastasis Rev 25:435-457

50. Massague J (2008) TGF-beta in Cancer. Cell 134:215-230 